\title{
Siderophore-mediated iron uptake in Saccharomyces cerevisiae: the SIT1 gene encodes a ferrioxamine $B$ permease that belongs to the major facilitator superfamily
}

\author{
Emmanuel Lesuisse, Monique Simon-Casteras and Pierre Labbe
}

Author for correspondence: Emmanuel Lesuisse. Tel: +33144278173 . Fax: +33144275716 e-mail: lesuisse $\left(a^{i j m}\right.$.jussieu.fr

Laboratoire de Biochimie des Porphyrines, Institut J. Monod, Tour 43, Université Paris 7, 2 Place Jussieu, 75251 Paris cedex 05, France

\begin{abstract}
Uptake of iron from various siderophores by a $\Delta$ fet $3 \Delta$ fet 4 strain of Saccharomyces cerevisiae was investigated. The catecholate enterobactin and the hydroxamate coprogen were taken up by the cells by passive diffusion, whereas the hydroxamates ferrioxamine B (FOB) and ferricrocin (FC) were taken up via a high-affinity energy-dependent mechanism. The kinetics of FOB and FC uptake showed reciprocal competitive inhibition. The transport was regulated by iron availability, but was independent of the Aft1p and Mac1p transcriptional activators. Mutants affected in the transport of FOB were isolated. The transport of FC was not impaired in these mutants. Functional complementation of one mutant allowed the identification of the SIT1 gene (Siderophore Iron Iransport) encoding a putative permease belonging to the major facilitator superfamily. The Sit1 protein is probably a permease specific for the transport of ferrioxamine-type siderophores. The evidence suggests that the uptake of ferrichrome-type siderophores like FC involves other specific permease(s), although there seems to be a common handling of FOB and FC following their internalization by the cell.
\end{abstract}

Keywords: iron uptake, siderophore, yeast

\section{INTRODUCTION}

The principal mechanism of iron uptake in Saccharomyces cerevisiae begins with the reduction of ferric iron at the cell surface (Lesuisse et al., 1987; Dancis et al., 1990). The iron is then taken up by the cells, by either a low-affinity transport system involving the product of the FET4 gene (Dix et al., 1994) or a high-affinity transport system that depends on a permease-oxidase complex encoded by the FET 3 and FTR 1 genes (Ask with et al., 1994; Stearman et al., 1996). Another strategy commonly used by micro-organisms to take up iron involves the excretion of siderophores. These are chelating agents that form complexes with $\mathrm{Fe}^{3+}$ with very high complexation constants. Ferrisiderophore complexes are formed outside the cells and are then taken up by the micro-organisms via specific transport systems. Most known fungal siderophores are hydroxamates (reviewed by van der Helm \& Winkelmann, 1994). Unlike many other yeasts, $S$. cerevisiae excretes

Abbreviations: (D)FC, (desferri)-ferricrocin; (D)FOB, (desferri)ferrioxamine $B$. no siderophore. However, we have shown (Lesuisse \& Labbe, 1989) that it can use siderophores excreted by other micro-organisms and take up iron, at least from ferrioxamine B (FOB), via a non-reductive mechanism (which means that the iron-siderophore complex enters the cell without prior reduction). The ability of $S$. cerevisiae to use 'xenosiderophores' could constitute a selective advantage of great value for this yeast. Hydroxamate siderophores excreted by bacteria and actinomycetes are abundant in nature. Their concentrations in bulk soils can reach $0 \cdot 1 \mu \mathrm{M}$ (Powell et al., 1980), and these concentrations can be substantially higher $(5-10 \mu \mathrm{M})$ in rhizosphere soil or soil having high microbial activity (Crowley et al., 1987). The opportunistic strategy that consists of developing specific uptake systems for xenosiderophores is not restricted to S. cerevisiae. Crowley et al. (1987) showed that several plants, such as monocotyledonous grasses, have direct mechanisms for acquiring iron from a variety of microbial siderophores. The molecular steps by which siderophores are taken up by bacteria are well-known, but those of eukaryotes have not yet been elucidated. Siderophore uptake by bacteria is often specific and 
involves a number of proteins, whose production is negatively regulated by iron, but no transport proteins have yet been identified in fungi. The yeast $S$. cerevisiae is a very good model system for investigating reductive iron assimilation in the eukaryotic cell (Askwith \& Kaplan, 1998). We believe that it is also a good model system for studying non-reductive iron uptake from siderophores. This report describes the use of a $\Delta f e t 3 \Delta$ fet 4 double mutant to investigate the mechanisms by which $S$. cerevisiae takes up iron-siderophore complexes. This mutant lacks both the high-affinity and lowaffinity transport systems involved in reductive iron uptake.

\section{METHODS}

Strains, media and iron compounds. The following strains of S. cerevisiae were used: DEY1455 (MAT $\alpha$ ade 2 can1 his 3 leu2 trp1 ura3 gal), DEY1433 (MAT $\alpha$ ade2 his3 leu2 trp1 ura3 fet.3::HIS3 fet 4::LEU2), DDY4 (MATa ade6 can1 bis3 leu2 trp1 ura3 fet3-2:: HIS3 fet4-1::LEU2) (Dix et al., 1997), S1502B (MATa his3 leu2 trp1 ura3), S150-2Baft1 and S150-2Bmac1. Unless otherwise stated, cells were grown in complete YPG medium (Difco) or in minimal YNB-glucose media (Difco) containing the required amino acids and varjous iron sources (as siderophores or ferric citrate). Desferri-compounds were obtained as described by Wiebe \& Winkelmann (1975). When required, they were labelled with ${ }^{55} \mathrm{Fe}[50 \mathrm{mCi}(1850 \mathrm{MBq})$ $\mathrm{mg}^{-1}$ ]. Throughout this work, the name "(desferri)-ferrioxamine $B^{\prime}$ (DFOB) refers to the commercially available mesylate derivative Desferal (Ciba-Geigy).

Iron uptake experiments. Cells from an overnight culture in YPG $\left(\mathrm{OD}_{600} 0.5\right)$ were used to inoculate fresh YPG medium containing $200 \mu \mathrm{M}$ bathophenanthrolinedisulphonic acid (iron-deficient medium) or with no addition (iron-rich medium). The cells were grown for $6 \mathrm{~h}$ at $30^{\circ} \mathrm{C}$, washed with distilled water and suspended at a final $\mathrm{OD}_{600}$ of 3 in $50 \mathrm{mM}$ citrate buffer ( $\mathrm{pH} 6.5)$ containing $4 \%(\mathrm{w} / \mathrm{v})$ glucose. The cell suspension was incubated for $3 \mathrm{~min}$ at $30^{\circ} \mathrm{C}$ and iron was added as ${ }^{55} \mathrm{Fe}$-labelled siderophores $(0 \cdot 1-5 \mu \mathrm{M})$. Aliquots $(1 \mathrm{ml})$ were withdrawn at intervals and added to a 200 -fold excess of unlabelled siderophore. Aliquots were kept on ice until the cells were harvested by filtering through nitrocellulose filters. The cells were washed on the filter with about $20 \mathrm{ml}$ $50 \mathrm{mM}$ citrate buffer ( $\mathrm{pH} 6.5$ ) containing a 10-fold excess of unlabelled siderophore and $2 \%(w / v)$ EDTA. The iron taken up by the cells was measured by liquid scintillation counting.

Selection of mutants. Cells of the DEY 1433 strain ( $\Delta$ fet $3 \Delta$ fet 4 ) were subjected to EMS mutagenesis, with about $50 \%$ survival. The cells were then plated (about 500 colonies per plate) on YNB-glucose-agar medium containing $20 \mu \mathrm{M}$ ferric citrate and incubated for $3 \mathrm{~d}$. The colonies were replicated on YNBglucose-agar medium containing $100 \mu \mathrm{M}$ DFOB and on YNBglucose-agar medium containing ferric citrate $(20 \mu \mathrm{M})$. Colonies that grew on ferric-citrate-containing medium but not on DFOB-containing medium were selected for further analysis. The mutants were analysed genetically by classic methods.

Molecular biology techniques. Functional complementation of the mutants was carried out using a genomic library constructed from the $\Delta$ fet $3 \Delta f e t 4$ strain. The genomic DNA was digested with Sau3A to obtain fragments of mean size of $5-8 \mathrm{~kb}$. The DNA fragments ranging from 3 to $15 \mathrm{~kb}$ were purified from the agarose gel after electrophoresis. The fragments were then ligated to the multicopy vector YEp352 previously digested by BamHI. The mutants were transformed by the resulting genomic library, and the cells were plated onto YNB-glucose-agar medium containing $0.1 \mathrm{mM}$ DFOB. The colonies that grew on such medium were transferred to YPG. agar medium and replica-plated onto YPG-agar medium containing 5-fluoroorotic acid (100 $\left.\mathrm{mg} \mathrm{l}^{-1}\right)$ to select cells that had lost the YEp352 (URA3) plasmid. The colonies were then tested for recovery of the mutant phenotype (did not grow on DFOB-containing medium). This selection step on 5fluoroorotic acid was important, since most of the clones that grew on DFOB-containing medium after transformation by the genomic library were revertants. The plasmid DNA was extracted from the clones of interest and amplified in Escherichia coli. The plasmid inserts were then analysed by sequencing and restriction studies. The YEL065w ORF (SIT1 gene) was disrupted using a disruption plasmid constructed from the YEp352 vector bearing a $4 \mathrm{~kb}$ fragment including the SIT1 ORF. A $300 \mathrm{bp}$ fragment in the middle of the ORF was removed by $\mathrm{Xhol} /$ BstXI digestion, and the $\operatorname{kanM}$ gene was ligated (blunt end) to the vector. The products of Puull digestion were used to transform the $\Delta$ fet $3 \Delta f e t 4$ strain and its wild-type counterpart. The disruptants were selected on YPGagar plates containing $200 \mathrm{mg}$ geneticin $\mathrm{I}^{-1}(\mathrm{G} 418)$. PCR methods were used to check the correct knock-out of the SITI gene.

\section{RESULTS AND DISCUSSION}

A strain of S. cerevisiae with disrupted FET3 and FET4 genes cannot take up iron by either the high-affinity or low-affinity reductive transport systems (Dix et al., 1997). This strain did not, therefore, grow on minimal medium (YNB-glucose, containing about $1 \mu \mathrm{M}$ iron), unless a large excess of iron [20 $\mu \mathrm{M} \mathrm{Fe}$ (III) citrate] was added to the medium (not shown). We have previously reported (Lesuisse \& Labbe, 1989) that $S$. cerevisiae has a non-reductive transport system with high affinity for FOB. When present at low concentrations (micromolar) this siderophore was taken up by the cells via a highaffinity transport system without prior reductive dissociation of the complex. Haem was required for siderophore dissociation within the cell (Lesuisse \& Labbe, 1989). Thus, we tested whether the addition of DFOB to a minimal medium would allow a $\Delta$ fet $3 \Delta f e t 4$ mutant to grow. We also tested the effect of two other hydroxamate-type siderophores [desferri-coprogen and desferri-ferricrocin (DFC)], and of one catecholate-type siderophore (desferri-enterobactin). All the siderophores tested led to growth of the cells on minimal medium (Fig. 1). Other iron chelators like salicylhydroxamic acid and dihydroxybenzoic acid did not restore cell growth (Fig. 1). The growth-promoting effect of the siderophores tested could be due to it being easier for iron to diffuse into the cells when in the form of ferrisiderophores, rather than 'free'; alternatively, the ferrisiderophores could be taken up actively by the cells. The active uptake possibility seems more probable, since an active, specific transport has already been shown for FOB (Lesuisse \& Labbe, 1989). In addition, the greatest effect was obtained with DFC. This siderophore (belonging to the ferrichrome family) allowed full growth of the mutant $(\Delta f e t 3 \Delta f e t 4)$ cells at a 


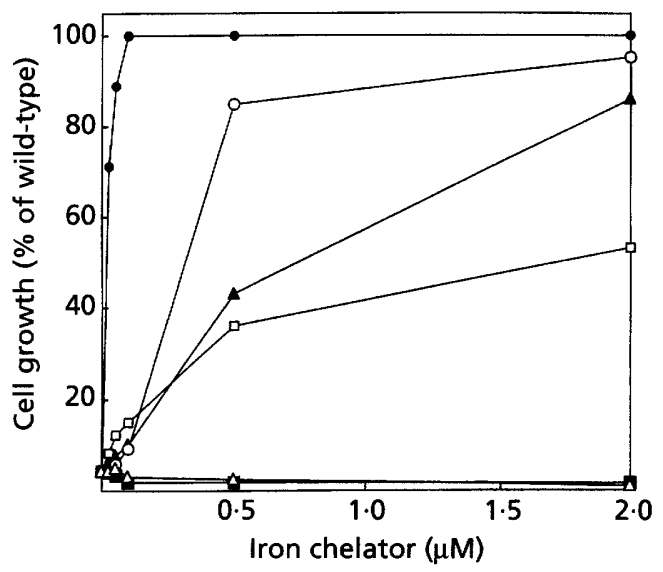

Fig. 1. Effect of various iron chelators on the growth of a $\Delta$ fet $3 \Delta$ fet4 strain on minimal (YNB) medium. Cells were inoculated at an $O D_{600}$ of 0.05 in YNB-glucose medium containing various concentrations of DFOB $(\boldsymbol{\Lambda})$, desferrienterobactin (O), DFC (O), desferri-coprogen ( $\square$ ), salicylhydroxamic acid $(\square)$ or dihydroxybenzoic acid $(\triangle)$. The optical density of the cultures was measured after $16 \mathrm{~h}$ growth and compared to the $O_{600}$ of a reference culture (wild-type cells on YNB-glucose without addition). The $O_{600}$ of the reference culture was 3 . The mutant cells did not grow in YNBglucose alone (not represented).

Table 1. Iron uptake from various iron sources by a $\Delta f e t 3 \Delta f e t 4$ strain and its wild-type parent

Iron uptake (pmol $\mathrm{min}^{-1}$ per $\mathrm{OD}_{600}$ unit) by resting cells previously grown for $6 \mathrm{~h}$ in iron-deficient medium was measured. The concentration of iron in the assay was $1 \mu \mathrm{M}$ in each case. Values represent means $\pm S E$ from three experiments.

\begin{tabular}{|lll|}
\hline Iron source & $\boldsymbol{\Delta}$ fet $\mathbf{3} \boldsymbol{\Delta} \boldsymbol{f e t} 4$ & Wild-type \\
\hline $\mathrm{Fe}(\mathrm{III})$ citrate & $0 \cdot 05 \pm 0 \cdot 02$ & $1 \cdot 80 \pm 0 \cdot 20$ \\
$\mathrm{Fe}(\mathrm{II})$ ascorbate & $0 \cdot 07 \pm 0 \cdot 01$ & $2 \cdot 30 \pm 0 \cdot 30$ \\
FOB & $2 \cdot 20 \pm 0 \cdot 40$ & $1 \cdot 90 \pm 0 \cdot 10$ \\
Enterobactin & $1 \cdot 40 \pm 0 \cdot 30$ & $2 \cdot 50 \pm 0 \cdot 50$ \\
Coprogen & $0 \cdot 90 \pm 0 \cdot 10$ & $1 \cdot 00 \pm 0 \cdot 05$ \\
FC & $6 \cdot 30 \pm 0 \cdot 40$ & $5 \cdot 20 \pm 0 \cdot 30$ \\
\hline
\end{tabular}

concentration of only $0 \cdot 1 \mu \mathrm{M}$ (Fig. 1), suggesting that there is a very high affinity uptake mechanism. We measured the rate of iron uptake from various iron sources in resting wild-type and $\Delta f e t 3 \Delta f e t 4$ cells, and found that $\mathrm{FOB}$, ferricrocin (FC), enterobactin and coprogen were all taken up efficiently by cells of both strains (Table 1). As expected, ferric citrate and ferrous ascorbate were not taken up by the $\Delta f e t 3 \Delta f e t 4$ strain (Table 1), demonstrating that the iron uptake from the siderophores was not due to a reductive mechanism.

The dependence on an energy source for transport and the kinetic characteristics of the transport were determined for each siderophore (Figs 2 and 3). The uptake of enterobactin was glucose-independent (not shown). This siderophore did not inhibit the uptake of FOB (Fig. 2a), and concentration-dependent uptake yielded a straight line (Fig. 3b). These data indicate that simple diffusion is the underlying principle of enterobactin iron transport. The same was true for coprogen (Figs $2 \mathrm{~b}$ and $3 \mathrm{~d}$ ). As previously shown (Lesuisse $\&$ Labbe, 1989), the uptake of FOB was glucose-dependent with saturation kinetics enabling the determination of $K_{\mathrm{m}}$ (about $0.5 \mu \mathrm{M}$ ) and $V_{\max }$ (about $2 \mathrm{pmol} \mathrm{min}^{-1}$ per $\mathrm{OD}_{600}$ unit) (Fig. 3a). The uptake of $\mathrm{FC}$ was also highly dependent on glucose (not shown) and showed saturation kinetics $\left(K_{\mathrm{m}}=0.2 \mu \mathrm{M} ; V_{\max }=7 \mathrm{pmol} \mathrm{min}^{-1}\right.$ per $\mathrm{OD}_{600}$ unit; Fig. 3c). FC strongly and competitively inhibited the uptake of FOB (Fig. 2c). Our data clearly indicate that both FOB and FC are taken up by the cells via energy-dependent, high-affinity mechanisms. The finding of competition for the transport of these two siderophores could mean that: (i) both siderophores are taken up by. the same transport system, including recognition and translocation; (ii) each siderophore is recognized by a separate receptor before their common transport; (iii) both siderophores are recognized by the same receptor protein prior to their separate transport; (iv) there is a common step in the handling of both siderophores downstream from their separate recognition and translocation (Fig. 4). A shared transport system functioning with two different siderophore receptors has been proposed for the uptake of coprogens and ferrichromes by Neurospora crassa (Winkelmann \& Huschka, 1987).

We investigated whether the siderophore-mediated iron uptake system was regulated in the same way as the reductive transport system. Aft1p and Mac1p mediate the transcriptional control of the genes involved in reductive iron uptake in response to iron (Aft1p) and copper (Mac1p) availability (Yamaguchi-Iwai et al., 1996; Georgatsou \& Alexandraki, 1994). The reductive transport system is also regulated to some extent at the post-transcriptional level, because the copy number of YAP genes, which are involved in the cell response to oxidative stress, affects ferrireductase activity but not the transcription of FRE genes (Lesuisse et al., 1995). $\mathrm{Cd}^{2+}$ ions also strongly inhibit ferrireductase activity (Lesuisse et al., 1995) by promoting degradation of Fre1p (unpublished data). The siderophore-mediated transport system of $S$. cerevisiae was also regulated by iron availability (Table 2), but it did not depend on the Aft $1 p$ transcription factor. The aft 1 mutant had very low rates of ferrous iron uptake in all the growth conditions tested, whereas the rate of FC uptake by the same mutant was higher than that of the wild-type (Table 2). This difference is probably due to the aft 1 mutant being constitutively iron-deficient, because the reductive iron uptake pathway is repressed in this mutant. The independent siderophore uptake pathway, which is also regulated by iron, was presumably induced to some extent in the aft 1 mutant. Similarly, the 


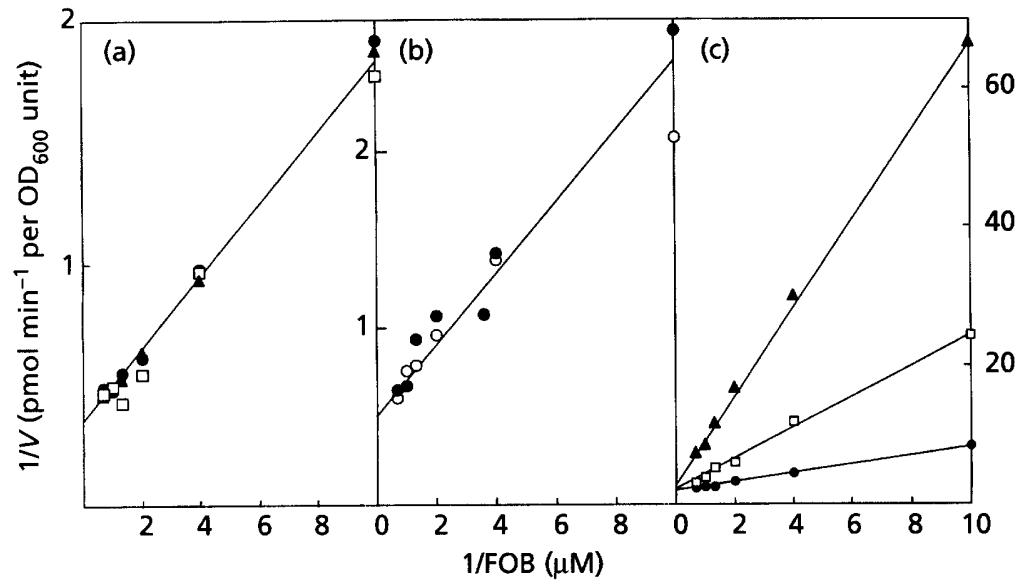

Fig. 2. Effect of enterobactin (a), coprogen (b) and FC (c) on the uptake rate of FOB at various concentrations. We measured FOB uptake by resting cells of the $\Delta$ fet $3 \Delta$ fet 4 strain previously grown for $6 \mathrm{~h}$ in irondeficient medium. The uptake of ${ }^{55} \mathrm{Fe}$ labelled FOB was measured in the presence or absence of unlabelled siderophores at various concentrations. (a) 9 , No addition; $\square, 1 \mu \mathrm{M}$ enterobactin; $\Delta, 5 \mu \mathrm{M}$ enterobactin. (b) No addition; O, $1 \mu \mathrm{M}$ coprogen. (c) 0 , No addition; $\square, 0.5 \mu \mathrm{M} \mathrm{FC;} \Delta, 2 \mu \mathrm{M}$ FC.

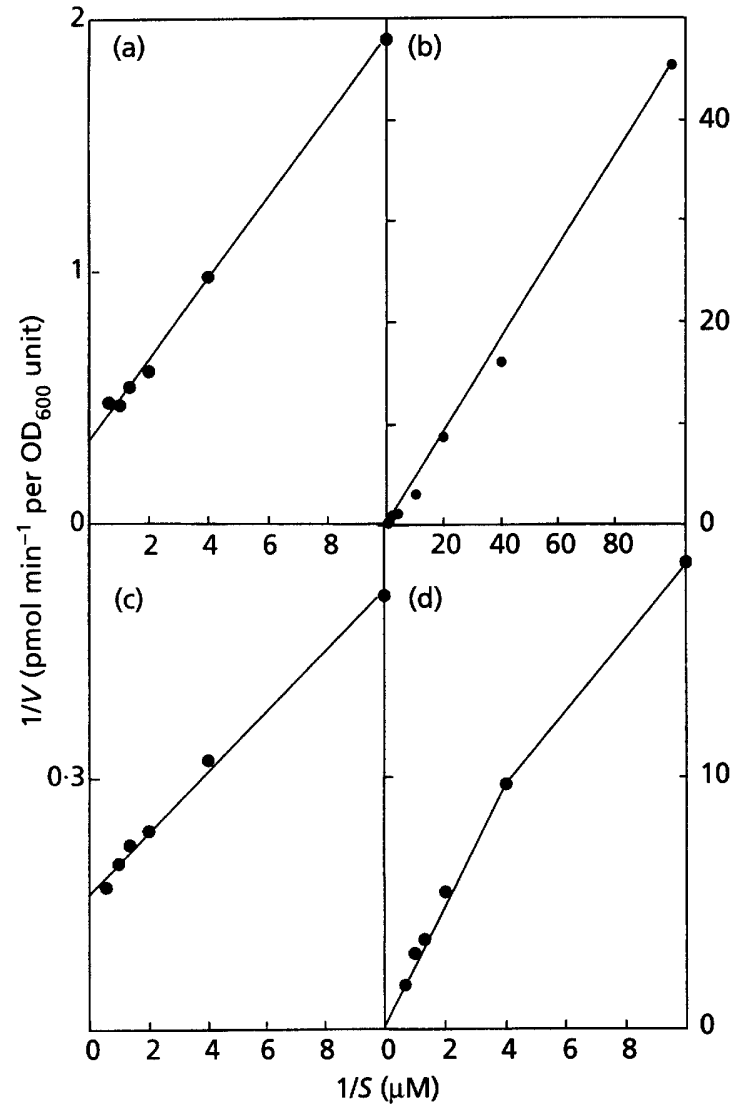

Fig. 3. Concentration-dependent uptake of FOB (a), enterobactin (b), FC (c) and coprogen (d) by cells of the $\Delta$ fet $3 \Delta$ fet4 strain. Uptake kinetics were measured with resting cells previously grown for $6 \mathrm{~h}$ in iron-deficient medium. Results are from one representative experiment.

chelation of copper by bathocuproin sulfonate during growth resulted in lower rates of ferrous iron uptake but not of FC uptake by resting cells (Table 2). Thus, although copper is required for ferrous iron uptake (see
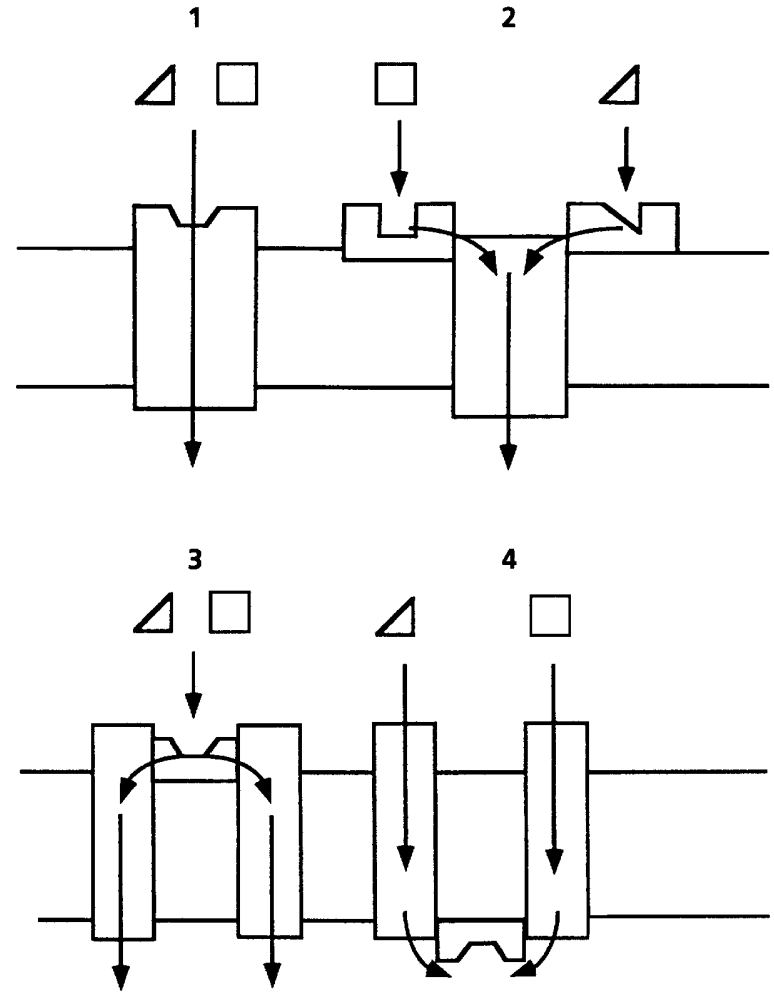

Fig. 4. Models of siderophore uptake in S. cerevisiae. Two types of siderophore (ferrioxamine-type and ferrichrome-type) are shown by squares and triangles. (1) Both siderophores are taken up by the same transport system, including recognition and translocation. (2) Both siderophores are recognized by separate receptors before common transport. (3) Both siderophores are recognized by the same receptor protein prior to separate transport. (4) There is a common step in the handling of both siderophores downstream to their separate recognition and translocation.

the role of Fet3p, Stearman et al., 1996), it does not seem to affect the uptake of siderophores. The presence of non-toxic concentrations of $\mathrm{Cd}^{2+}$ ions in the growth medium inhibited the uptake of ferrous iron and 
Table 2. Iron uptake by an aft1 strain and its wild-type parental strain grown under various conditions

Measurements were made of iron uptake $\left(\mathrm{pmol} \mathrm{min}^{-1}\right.$ per $\mathrm{OD}_{600}$ unit) in either $0.5 \mu \mathrm{M} \mathrm{FC}$ or $0.5 \mu \mathrm{M}$ ferrous ascorbate $\left(\mathrm{Fe}^{2+}\right)$ by resting cells previously grown for $6 \mathrm{~h}$ in YPG medium containing: (1) no additions; (2) $200 \mu \mathrm{M}$

bathophenanthrolinedisulphonic acid (BPS); (3) $200 \mu \mathrm{M}$ bathocuproin disulphonic acid (BCS); (4) $200 \mu \mathrm{M}$ ferrozine; (5)

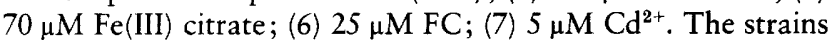
used were S150-2B and S150-2Baft1. ND, Not determined. These data are from one representative experiment.

\begin{tabular}{|c|c|c|c|c|}
\hline \multirow{2}{*}{ Growth conditions } & \multicolumn{2}{|c|}{ Wild-type } & \multicolumn{2}{|c|}{ aft1 mutant } \\
\hline & FC & $\mathrm{Fe}^{2+}$ & FC & $\mathrm{Fe}^{2+}$ \\
\hline YPG & $0 \cdot 70$ & $0 \cdot 40$ & $1 \cdot 00$ & $0 \cdot 10$ \\
\hline $\mathrm{YPG}+\mathrm{BPS}$ & $2 \cdot 20$ & $1 \cdot 40$ & $3 \cdot 20$ & $0 \cdot 20$ \\
\hline YPG + BCS & $0 \cdot 70$ & $0 \cdot 10$ & $1 \cdot 10$ & 0.05 \\
\hline YPG + ferrozine & $1 \cdot 40$ & $1 \cdot 90$ & $2 \cdot 90$ & $0 \cdot 30$ \\
\hline$Y P G+F e(I I I)$ citrate & $0 \cdot 40$ & $0 \cdot 05$ & ND & ND \\
\hline$Y P G+F C$ & $0 \cdot 60$ & 0.05 & ND & ND \\
\hline $\mathrm{YPG}+\mathrm{Cd}^{2+}$ & $1 \cdot 80$ & $0 \cdot 10$ & ND & ND \\
\hline
\end{tabular}

stimulated the uptake of $\mathrm{FC}$ by the resting cells (Table 2). The uptake of FC was also independent of the MAC1 and YAP1/YAP2 gene products (data not shown). Thus, $S$. cerevisiae clearly has at least two high-affinity iron uptake systems that are regulated independently. The Fet $3 p / F$ tr1p-dependent system is mostly regulated via transcriptional control mediated by Aft1p (YamaguchiIwai et al., 1996), whereas the mechanisms by which the siderophore uptake system is regulated by iron availability are unknown. Siderophore uptake was inhibited less than reductive uptake by excess iron in the growth medium (in the form of ferric citrate or FC) (Table 2).

We obtained two mutants (from about 50000 colonies screened) by EMS mutagenesis of the $\Delta$ fet $3 \Delta f e t 4$ strain that could not grow on YNB-glucose medium containing DFOB (Fig. 5). These mutants were deficient in both reductive iron transport and $\mathrm{FOB}$ uptake. Both mutants had the same $K_{\mathrm{m}}$ value as the parental strain (about $0.5 \mu \mathrm{M})$ for the uptake of FOB, but $V_{\max }$ values 10 times lower $\left(0 \cdot 2-0 \cdot 3\right.$ pmol min ${ }^{-1}$ per $\mathrm{OD}_{600}$ unit). However, uptake of FC by the mutants was similar to that for the parental strain (data not shown). The uptake of both FOB and FC by the mutant strains was still regulated by iron availability during growth, as in the parental strain. Thus, both mutants probably lack some component of the translocation system rather than of the regulation of transport.

We then focused on a single mutant, which had the most severely affected FOB transport. The mutant was crossed with a $\Delta$ fet $3 \Delta$ fet 4 strain of the opposite mating type, and the phenotype of the diploid strain and of haploid segregants was determined (Table 3). The mutation segregated $2: 2$, which strongly suggests that a single mutation was involved. We called the mutation responsible for the transport deficiency sit1-1 (siderophore iron transport). The rate of FOB transport by the SIT1/sit $1-1$ heterozygote was only $25 \%$ of that observed for the SIT1 parental haploid strain (Table 3). The $K_{\mathrm{m}}$ value for transport was, however, similar to that of the SIT1 and sit1-1 haploid strains (not shown). However, the slow rate of transport made it possible for the heterozygote to grow as well as the parental wild strain on minimal medium containing DFOB (not shown). This characteristic made it possible to screen a genomic library by functional complementation of the mutant. After transformation of the sit1-1 mutant by the $\Delta f e t 3 \Delta f e t 4$ genomic library, we identified three independent clones that had recovered plasmid-dependent transport of FOB. A unique ORF, YEL065w, was common to the three plasmid inserts (size $3-8 \mathrm{~kb}$ ). The product of the YEL065 $\mathrm{w}$ ORF belongs to the major facilitator superfamily (MFS), and has been classified among the multidrug permease homologues for which no substrate is known (Nelissen et al., 1997). The protein has 628 amino acids and 12 predicted transmembrane spans and is very similar to the products of YHL040c and YHLO47c, two other putative permeases for which there is no known substrate (Nelissen et al., 1997). No significant similarity was found between the product of YEL065w and known bacterial siderophore transport protein (not shown). The gene promoter does not show the consensus sequence for Aft1p transcriptional regulation. The YEL065 $\mathrm{w}$ ORF was disrupted in both the wild-type and the $\Delta$ fet $3 \Delta f e t 4$ strain. As shown in Table 4, the $\Delta$ fet $3 \Delta$ fet 4 strain disrupted for the YEL065w ORF had no FOB transport activity, while the uptake of FC was only slightly affected. There was still residual iron uptake from FOB in the disrupted wildtype strain, which was probably due to reductive iron uptake. As YEL065w encodes a putative permease, it is reasonable to assume that its product, Sit 1 , is the major protein involved in the trans-plasma membrane translocation of FOB. Since there was competitive inhibition for the uptake of FOB and FC (Fig. 2), and since disruption of the SIT1 gene led to defective transport only of FOB (Table 4), there cannot be a common recognition and transfer process for both FOB and FC (Fig. 4). To determine whether the competitive effect occurred upstream or downstream to translocation, the competitive effect of $\mathrm{FOB}$ on the uptake of $\mathrm{FC}$ was measured in the $\Delta f e t 3 \Delta f e t 4$ strain and in the $\Delta f e t 3 \Delta f e t 4$ $\Delta$ sit1 strain. FC uptake was inhibited by increasing concentrations of FOB in the SIT1 strain, but not in the sit1 mutant (Fig. 6). These data strongly suggest that the competitive effect in the uptake of FOB and FC occurred downstream to the translocation of the siderophores (see the model 4 in Fig. 4). This would mean that after a separate and specific recognition/translocation process through the plasma membrane, the ferrioxamine-type and the ferrichrome-type siderophores could share a common step in their intracellular processing. For example, both siderophores could be bound to the same chaperone protein after their translocation into the cell. 

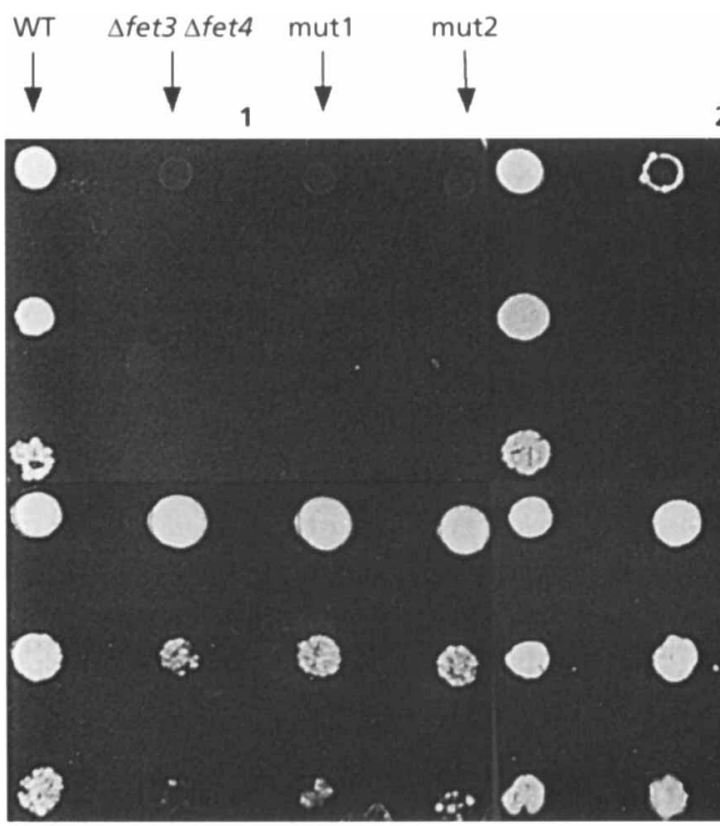

3

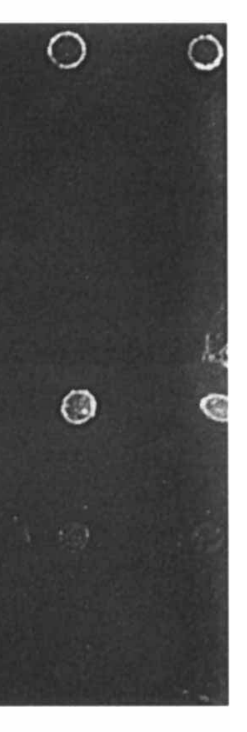

4
Fig. 5. Growth on YNB-glucose-agar medium of the wild-type strain, of the $\Delta$ fet $3 \Delta$ fet 4 strain and of two mutant strains obtained by EMS mutagenesis of the $\Delta$ fet $3 \Delta$ fet 4 strain. Additions to the medium: (1) none; (2) $25 \mu \mathrm{M} \mathrm{Fe}$ (III) citrate; (3) $50 \mu \mathrm{M}$ Fe(III) citrate; (4) $50 \mu \mathrm{M}$ DFOB. WT, Wildtype.

\section{Table 3. Uptake of FOB by various $\Delta$ fet $3 \Delta$ fet 4 strains}

Iron uptake (pmol $\min ^{-1}$ per $\mathrm{OD}_{600}$ unit) by resting cells previously grown for $6 \mathrm{~h}$ in iron-deficient medium was measured. The FOB concentration was $1 \mu \mathrm{M}$ in each case.

\begin{tabular}{|ll|}
\hline Strain/tetrad & Rate of FOB uptake \\
\hline SIT1 haploid strain & $1 \cdot 80$ \\
sit1 1 mutant & $0 \cdot 12$ \\
SIT1/sit1-1 heterozygote & $0 \cdot 47$ \\
Tetrad 1 & $0 \cdot 12 / 0 \cdot 15 / 1 \cdot 20 / 1 \cdot 40$ \\
Tetrad 2 & $0 \cdot 18 / 0 \cdot 09 / 1 \cdot 80 / 1 \cdot 30$ \\
Tetrad 3 & $0 \cdot 16 / 0 \cdot 15 / 1 \cdot 40 / 1 \cdot 70$ \\
Tetrad 4 & $0 \cdot 13 / 0 \cdot 12 / 1 \cdot 80 / 1 \cdot 80$ \\
\hline
\end{tabular}

Table 4. Uptake of FOB and FC by various strains

Iron uptake (pmol $\min ^{-1}$ per $\mathrm{OD}_{600}$ unit) by resting cells previously grown for $6 \mathrm{~h}$ in iron-deficient medium was measured. The FOB concentration was $1 \mu \mathrm{M}$ in each case. Values represent means \pm SE from three experiments.

\begin{tabular}{|c|c|c|}
\hline \multirow[t]{2}{*}{ Strain/genotype } & \multicolumn{2}{|c|}{ Rate of iron uptake } \\
\hline & FOB & FC \\
\hline SIT1 (wild-type) & $1 \cdot 40 \pm 0 \cdot 20$ & $2 \cdot 80 \pm 0 \cdot 20$ \\
\hline $\operatorname{SIT} 1(\Delta \mathrm{fet} 3 \Delta \mathrm{fet} 4)$ & $1 \cdot 90 \pm 0 \cdot 20$ & $3 \cdot 50 \pm 0 \cdot 40$ \\
\hline$\Delta s i t 1$ (wild-type) & $0 \cdot 05$ & $2 \cdot 40 \pm 0 \cdot 30$ \\
\hline$\Delta s i t 1(\Delta f e t 3 \Delta f e t 4)$ & 0.00 & $2 \cdot 90 \pm 0 \cdot 40$ \\
\hline YEp352-SIT1 $(\Delta f e t 3 \Delta f e t 4 \Delta s i t 1)$ & $3 \cdot 60 \pm 0 \cdot 40$ & $3 \cdot 40 \pm 0 \cdot 20$ \\
\hline
\end{tabular}

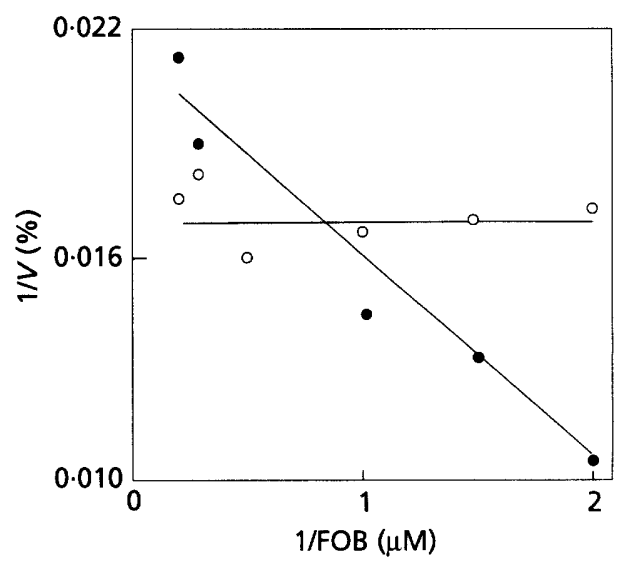

Fig. 6. Effect of FOB concentration on the rate of FC uptake by a $\Delta$ fet $3 \Delta$ fet4 strain (O) and a $\Delta$ fet $3 \Delta$ fet4 $\Delta$ sit1 strain (O). FC concentration was $0.25 \mu \mathrm{M}$. Results are expressed as percentage of the rate of FC uptake measured in the $\Delta$ fet $3 \Delta$ fet 4 strain without added FOB $\left(100 \%=2 \cdot 1 \mathrm{pmol} \min ^{-1}\right.$ per $\mathrm{OD}_{600}$ unit $)$.

\section{General conclusions}

In addition to the reductive mechanism of iron uptake, S. cerevisiae has at least two other high-affinity transport systems for non-reductive siderophore assimilation, one for ferrioxamine-type siderophores and another for ferrichrome-type siderophores. Sit 1 was identified as the ferrioxamine permease. It is tempting to speculate that the Sit 1 homologues could be permeases involved in the transport of other siderophore families. On the basis of their great similarity, Nelissen et al. (1997) suggested 
that these proteins should have similar functions. The energetics of siderophore transport in S. cerevisiae remain unclear. The Sit1 protein and its homologues have no ATP-binding cassette motif. On the basis of bacterial protein homologies, Goffeau et al. (1997) suggested that the members of this MFS cluster could function as $\mathrm{H}^{+}{ }_{\text {in }} / \mathrm{DRUG}_{\text {out }}$ antiports. The Sit 1 protein is responsible for importing of $\mathrm{FOB}$; we are currently trying to determine whether the import of FOB could depend on ATPase-dependent proton excretion by the cells.

Despite the fact that $S$. cerevisiae does not itself produce any siderophore, the non-reductive uptake system for xenosiderophores in this yeast has the same characteristics as the siderophore uptake systems of siderophore-producing micro-organisms: regulation by iron, energy-dependence, high specificity, high affinity, complex processing of the internalized siderophores. We previously showed that haem-deficient cells are still able to take up FOB, but can no longer dissociate iron from the ferrisiderophore (Lesuisse \& Labbe, 1989). This strongly suggests that the haem-dependent reduction of the siderophore is required for the dissociation of iron from the complex and its subsequent use. Many yeastlike organisms could have two types of high-affinity iron transport systems, one reductive and one non-reductive. Inducible reductive uptake systems have now been recognized in numerous fungi, including siderophore producers, such as species of the following genera: Schizosaccharomyces, Pichia, Hyphopichia, Candida, Kluyveromyces, Endomyces, Yarrowia, Ustilago, Rhodotorula (Emery, 1987; Roman et al., 1993; Lesuisse et al., 1995; Morrissey et al., 1996; E. Lesuisse, unpublished results). In $S$. cerevisiae, the non-reductive and the reductive transport systems seem to be regulated independently. The physiological significance of such a dual regulatory mechanism affecting iron uptake is questionable. We are currently trying to determine whether the reductive and non-reductive uptake systems can each be induced/repressed under definite physiological conditions that cannot be separated under standard laboratory growth conditions.

\section{ACKNOWLEDGEMENTS}

We thank Dr G. Winkelmann and Dr D. Expert for kindly donating siderophores and $\mathrm{Dr} \mathrm{D}$. Eide for the yeast strains. This work was supported by grants from CNRS and Université Paris 7. We thank Dr O. Parkes for checking the manuscript.

\section{REFERENCES}

Askwith, C. \& Kaplan, J. (1998). Iron and copper transport in yeast and its relevance to human disease. Trends Biochem Sci 23, 135-138.

Askwith, C., Eide, D., VanHo, A., Bernard, P. S., Li, L., DavisKaplan, S., Sipe, D. M. \& Kaplan, J. (1994). The FET3 gene of $S$. cerevisiae encodes a multicopper oxidase required for ferrous iron uptake. Cell 76, 403-410.

Crowley, D. E., Reid, C. P. P. \& Szaniszlo, P. J. (1987). Microbial siderophores as iron sources for plants. In Iron Transport in Microbes, Plants and Animals, pp. 375-386. Edited by G. Winkelmann, D. van der Helm \& J. B. Neilands. Weinheim \& New York: VCH.

Dancis, A., Klausner, R. D., Hinnebusch, A. G. \& Barriocanal, J. G. (1990). Genetic evidence that ferric reductase is required for iron uptake in Saccharomyces cerevisiae. Mol Cell Biol 10, 2294-2301.

Dix, D. R., Bridgham, J. T., Broderius, M. A., Byersdorfer, C. A. \& Eide, D. J. (1994). The FET4 gene encodes the low affinity $\mathrm{Fe}(\mathrm{II})$ transport protein of Saccharomyces cerevisiae. J Biol Chem 269, 26092-26099.

Dix, D., Bridgham, J., Broderius, M. \& Eide, D. (1997). Characterization of the FET4 protein of yeast. Evidence for a direct role in the transport of iron. J Biol Chem 272, 11770-11777.

Emery, T. (1987). Reductive mechanisms of iron assimilation. In Iron Transport in Microbes, Plants and Animals, pp. 235-250. Edited by G. Winkelmann, D. van der Helm \& J. B. Neilands. Weinheim \& New York: VCH.

Georgatsou, E. \& Alexandraki, D. (1994). Two distinctly regulated genes are required for ferric reduction, the first step of iron uptake in Saccharomyces cerevisiae. Mol Cell Biol 14, 3065-3073.

Goffeau, A., Park, J., Paulsen, I. T., Jonniaux, J. L., Dinh, T., Mordant, P. \& Saier, M. H. (1997). Multidrug-resistant transport proteins in yeast: complete inventory and phylogenetic characterization of yeast open reading frames with the major facilitator superfamily. Yeast 13, 43-54.

van der Helm, D. \& Winkelmann, G. (1994). Hydroxamates and polycarboxylates as iron transport agents (siderophores) in fungi. In Metal Ions in Fungi, pp. 39-98. Edited by G. Winkelmann \& D. R. Winge. New York, Basel \& Hong Kong: Marcel Dekker.

Lesuisse, E. \& Labbe, P. (1989). Reductive and non-reductive mechanisms of iron assimilation by the yeast Saccharomyces cerevisiae. J Gen Microbiol 135, 257-263.

Lesuisse, E. \& Labbe, P. (1995). Effects of cadmium and of YAP1 and CAD1/YAP2 genes on iron metabolism in the yeast Saccharomyces cerevisiae. Microbiology 141, 2937-2943.

Lesuisse, E., Raguzzi, F. \& Crichton, R. (1987). Iron uptake by the yeast Saccharomyces cerevisiae: involvement of a reduction step. J Gen Microbiol 133, 3228-3236.

Lesuisse, E., Casteras-Simon, M. \& Labbe, P. (1995). Ferrireductase activity in Saccharomyces cerevisiae and other fungi : colorimetric assays on agar plates. Anal Biochem 226, 375-377.

Morrissey, J. A., Williams, P. H. \& Cashmore, A. M. (1996). Candida albicans has a cell-associated ferric-reductase activity which is regulated in response to levels of iron and copper. Microbiology 142, 485-492.

Nelissen, B., De Wachter, R. \& Goffeau, A. (1997). Classification of all putative permeases and other membrane plurispanners of the major facilitator superfamily encoded by the complete genome of Saccharomyces cerevisiae. FEMS Microbiol Rev 21, 113-134.

Powell, P. E., Cline, G. R., Reid, C. P. P. \& Szanislo, J. (1980). Occurrence of hydroxamate siderophore iron chelates in soils. Nature 287, 833-835.

Roman, D. G., Dancis, A., Anderson, G. I. \& Klausner, R. D. (1993). The fission yeast ferric reductase gene frp $1+$ is required for ferric iron uptake and encodes a protein that is homologous to the gp91phox subunit of the human NADPH phagocyte oxidoreductase. Mol Cell Biol 13, 4342-4350. 
Stearman, R., Yuan, D. S., Yamaguchi-Iwai, Y., Klausner, R. D. \& Dancis, A. (1996). A permease-oxidase complex involved in highaffinity iron uptake in yeast. Science 271, 1552-1557.

Wiebe, C. \& Winkelmann, G. (1975). Kinetic studies on the specificity of chelate-iron uptake in Aspergillus. J Bacteriol 123, $837-842$.

Winkelmann, G. \& Huschka, H. G. (1987). Molecular recognition and transport of siderophores in fungi. In Iron Transport in Microbes, Plants and Animals, pp. 317-336. Edited by G.
Winkelmann, D. van der Helm \& J. B. Neilands. Weinheim \& New York: VCH.

Yamaguchi-Iwai, Y., Stearman, R., Dancis, A. \& Klausner, R. D. (1996). Iron-regulated DNA binding by the AFT1 protein controls the iron regulon in yeast. EMBO J 15, 3377-3384.

Received 20 July 1998; revised 7 September 1998; accepted 9 September 1998. 